\title{
A New Method Applied to the Quadrilateral Membrane Element with Vertex Rigid Rotational Freedom
}

\author{
Xiaowei Gao, Yunfei Liu, and Jun Lv \\ School of Aeronautics and Astronautics, State Key Laboratory of Structural Analysis for Industrial Equipment, \\ Dalian University of Technology, Dalian 116024, China
}

Correspondence should be addressed to Jun Lv; lvjun@dlut.edu.cn

Received 24 November 2015; Revised 20 January 2016; Accepted 26 January 2016

Academic Editor: Francesco Tornabene

Copyright (C) 2016 Xiaowei Gao et al. This is an open access article distributed under the Creative Commons Attribution License, which permits unrestricted use, distribution, and reproduction in any medium, provided the original work is properly cited.

In order to improve the performance of the membrane element with vertex rigid rotational freedom, a new method to establish the local Cartesian coordinate system and calculate the derivatives of the shape functions with respect to the local coordinates is introduced in this paper. The membrane elements with vertex rigid rotational freedom such as GQ12 and GQ12M based on this new method can achieve higher precision results than traditional methods. The numerical results demonstrate that the elements GQ12 and GQ12M with this new method can provide better membrane elements for flat shell elements. Furthermore, this new method presented in this paper offers a new approach for other membrane elements used in flat shell element to improve the computing accuracy.

\section{Introduction}

The finite element method has been used for solving problems in different fields of engineering. For now, a large number of different finite elements have been developed and the shell element is one of these elements to solve the multiscale problems $[1,2]$. The shell element can be categorized into two types: curved shell element and flat shell element [3]. The curved shell element can be used to make a good description of the geometrical shape of the shell structure, so it is with a good calculation precision. But the formula expressions of the curved shell element are very complicated so it is limited in practical application. In this case, the flat shell element is becoming more attractive in deriving efficient numerical accuracy and its concise theory due to its superposition theory of the membrane element and plate bending element [4].

The membrane elements are among the simplest elements to develop, which are used for analyzing structures subjected to in-plane forces. The membrane elements are usually used to model the behavior of shear wall, stiffened sheet construction, and membrane action in shells. Some plane elements can be considered as membrane elements, such as the CST (constant strain triangle) element and the fournode isoparametric quadrilateral plane element (QUAD4) [5]. In finite element methods, many plate bending elements also have been developed. Bazeley et al. [6] developed the confirming and nonconfirming plate bending elements in 1966. Batoz et al. [7] developed three types of plate bending elements, that is, the DKT element, the HSM element, and the SRI element for the analysis of plates and shells in 1980. In 1982, the quadrilateral plate bending element (DKQ) was formulated by Batoz and Tahar [8] based on the DKT element. Other plate bending elements have been developed in the following years [9-12].

The flat shell elements are developed by combining membrane elements containing two in-plane translational degrees of freedom and plate bending elements containing two rotational degrees of freedom and one out of plane translational degree of freedom. Since the in-plane rotational degrees of freedom are not included, the null values for the inplane rotational degrees of freedom will lead to singularity in structure stiffness matrix if all the elements are coplanar. The simplest method adopted to remove the singularity is to add the in-plane rotational degrees of freedom to the membrane elements, which can also improve their performance [5]. 


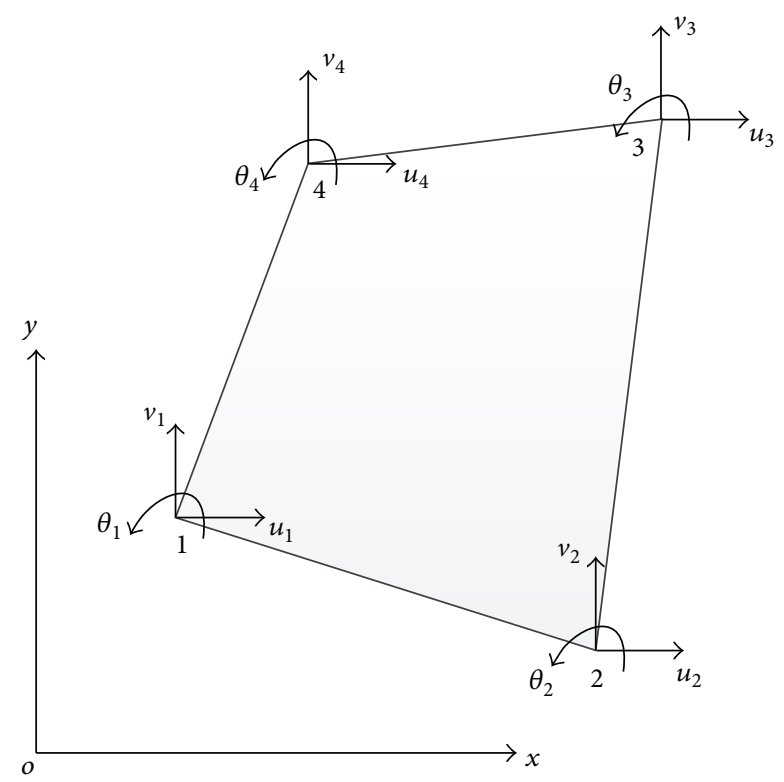

FIGURE 1: The quadrilateral membrane element.

After several triangular elements [13, 14] with vertex rigid rotational freedom are proposed, the quadrilateral membrane elements including corner rotations have been developed by Allman [15] and MacNeal and Harder [16] as well. And then, numerous researches focused on membrane elements with drilling degrees of freedom have been accomplished, such as the models proposed by Iura and Atluri [17], Cazzani and Atluri [18], Piltner and Taylor [19], Geyer and Groenwold [20], Groenwold et al. [21], Choi et al. [22], Kugler et al. [23], and Cen et al. [24]. In 1994, two new arbitrary quadrilateral membrane elements called GQ12 and GQ12M with vertex rotation were proposed by Long and $\mathrm{Xu}$ [25], resulting in more reasonable compatible conditions between adjoining elements and a more simple formulation.

In order to further improve the computing accuracy of the elements GQ12 and GQ12M, a new method for establishing the local Cartesian coordinate system and calculating the derivatives of the shape function with respect to the local coordinates is presented in this paper. This new method can be applied to the membrane elements which may provide a constituent part for flat shell elements. The elements GQ12 and GQ12M have more accurate numerical results compared to the bilinear quadrilateral element Q4 $[3,25]$. In this paper, the applications of the new method to the elements GQ12 and GQ12M were proposed to examine the performance of this new method described in this paper.

\section{The Membrane Elements GQ12 and GQ12M}

A quadrilateral membrane element with rotational degree of freedom (shown in Figure 1) was proposed in [25]. by

The nodal displacement vector over this element is given

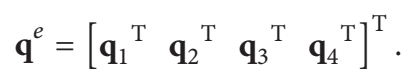

The freedoms at each node are

$$
\mathbf{q}_{i}=\left[\begin{array}{lll}
u_{i} & v_{i} & \theta_{i}
\end{array}\right]^{\mathrm{T}}, \quad(i=1,2,3,4)
$$

in which $u_{i}$ and $v_{i}$ are the translations and $\theta_{i}$ is the additional rotation at each corner. A membrane element is usually located in a three-dimensional space, so its orientation may be in any directions. For convenience, the membrane element is commonly studied in the local Cartesian coordinate system represented by $\left(x^{\prime}, y^{\prime}, z^{\prime}\right)$ and located on the membrane element. Once a quantity is formed in the local system, it can be transformed to the global coordinate system.

In the local system, the element GQ12 is formulated by the displacement field which includes two parts as follows [25]:

$$
\mathbf{u}=\mathbf{u}^{0}+\mathbf{u}_{\theta}
$$

in which $\mathbf{u}^{0}$ is the conventional bilinear compatible displacement decided by the translations $\left[\begin{array}{ll}u_{i} & v_{i}\end{array}\right]^{\mathrm{T}}$ at the nodes and $\mathbf{u}_{\theta}$ is the additional displacement determined by the rigid rotation $\theta_{i}(i=1,2,3,4)$ at each node. The element GQ12 displacement can be described in the form of

$$
\mathbf{u}=\mathbf{u}^{0}+\mathbf{u}_{\theta}=\mathbf{N q}^{e}=\sum_{i=1}^{4} \mathbf{N}_{i} \mathbf{q}_{i}
$$

where $\mathbf{N}$ is the shape function matrix of element GQ12, which can be given in terms of

$$
\mathbf{N}_{i}=\left[\begin{array}{ccc}
N_{i}^{0} & 0 & N_{u \theta i} \\
0 & N_{i}^{0} & N_{v \theta i}
\end{array}\right], \quad(i=1,2,3,4)
$$

in which

$$
\begin{aligned}
& N_{i}^{0}=\frac{1}{4}\left(1+\xi_{i} \xi\right)\left(1+\eta_{i} \eta\right), \quad(i=1,2,3,4), \\
& \begin{aligned}
N_{u \theta i} & =\frac{1}{8}\left[\xi_{i}\left(1-\xi^{2}\right)\left(b_{1}+b_{3} \eta_{i}\right)\left(1+\eta_{i} \eta\right)\right. \\
& \left.+\eta_{i}\left(1-\eta^{2}\right)\left(b_{2}+b_{3} \xi_{i}\right)\left(1+\xi_{i} \xi\right)\right], \\
N_{\nu \theta i} & =-\frac{1}{8}\left[\xi_{i}\left(1-\xi^{2}\right)\left(a_{1}+a_{3} \eta_{i}\right)\left(1+\eta_{i} \eta\right)\right. \\
& \left.+\eta_{i}\left(1-\eta^{2}\right)\left(a_{2}+a_{3} \xi_{i}\right)\left(1+\xi_{i} \xi\right)\right], \quad(i=1,2,3,4),
\end{aligned}
\end{aligned}
$$

in which

$$
\begin{aligned}
& a_{1}=\frac{1}{4} \sum_{i=1}^{4} \xi_{i} x_{i}^{\prime}, \\
& a_{2}=\frac{1}{4} \sum_{i=1}^{4} \eta_{i} x_{i}^{\prime}, \\
& a_{3}=\frac{1}{4} \sum_{i=1}^{4} \xi_{i} \eta_{i} x_{i}^{\prime},
\end{aligned}
$$




$$
\begin{aligned}
& b_{1}=\frac{1}{4} \sum_{i=1}^{4} \xi_{i} y_{i}^{\prime}, \\
& b_{2}=\frac{1}{4} \sum_{i=1}^{4} \eta_{i} y_{i}^{\prime}, \\
& b_{3}=\frac{1}{4} \sum_{i=1}^{4} \xi_{i} \eta_{i} y^{\prime},
\end{aligned}
$$

where $\xi_{i}, \eta_{i}$ and $x_{i}^{\prime}, y_{i}^{\prime}(i=1,2,3,4)$ denote the intrinsic coordinates and the local Cartesian coordinates of the element nodes, respectively.

In the local system, the element stiffness matrix of GQ12 can be written as [25]

$$
\mathbf{K}_{\mathrm{GQ} 12}^{\prime e}=\iint_{A_{e}} \mathbf{B}^{\mathrm{T}} \mathbf{D B} t d A=\iint_{-1}^{1} \mathbf{B}^{\mathrm{T}} \mathbf{D B}|\mathbf{J}| t d \xi d \eta,
$$

where $t$ is the thickness of the element, $|\mathbf{J}|$ is the determinant of the Jacobian matrix, and $\mathbf{B}$ is the element strain matrix which can be expressed as

$$
\begin{aligned}
& \mathbf{B}=\left[\begin{array}{llll}
\mathbf{B}_{1} & \mathbf{B}_{2} & \mathbf{B}_{3} & \mathbf{B}_{4}
\end{array}\right], \\
& \mathbf{B}_{i}=\left[\begin{array}{cc}
\frac{\partial}{\partial x^{\prime}} & 0 \\
0 & \frac{\partial}{\partial y^{\prime}} \\
\frac{\partial}{\partial y^{\prime}} & \frac{\partial}{\partial x^{\prime}}
\end{array}\right]\left[\begin{array}{ccc}
N_{i}^{0} & 0 & N_{u \theta i} \\
0 & N_{i}^{0} & N_{v \theta i}
\end{array}\right] \\
& =\left[\begin{array}{ccc}
\frac{\partial N_{i}^{0}}{\partial x^{\prime}} & 0 & \frac{\partial N_{u \theta i}}{\partial x^{\prime}} \\
0 & \frac{\partial N_{i}^{0}}{\partial y^{\prime}} & \frac{\partial N_{v \theta i}}{\partial y^{\prime}} \\
\frac{\partial N_{i}^{0}}{\partial y^{\prime}} & \frac{\partial N_{i}^{0}}{\partial x^{\prime}} & \frac{\partial N_{u \theta i}}{\partial y^{\prime}}+\frac{\partial N_{v \theta i}}{\partial x^{\prime}}
\end{array}\right],
\end{aligned}
$$
[4]

In (9), D is the elasticity matrix and it can be expressed as

$$
\mathbf{D}=\frac{E}{1-\mu^{2}}\left[\begin{array}{ccc}
1 & \mu & 0 \\
\mu & 1 & 0 \\
0 & 0 & \frac{1-\mu}{2}
\end{array}\right]
$$

in which $E$ is the elastic modulus and $\mu$ is Poisson's ratio.

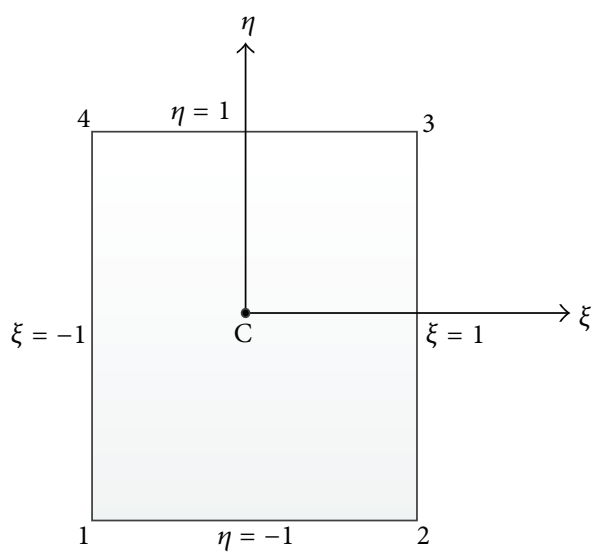

FIGURE 2: The adding bubble node C.

In order to further improve the precision of the calculation, the element GQ12M based on the element GQ12 was presented in [25] by Long and $\mathrm{Xu}$. The element GQ12M is formulated with an assumed displacement given by adding a bubble displacement $\widehat{\mathbf{u}}$ to GQ12, which is

$$
\mathbf{u}=\mathbf{u}^{0}+\mathbf{u}_{\theta}+\widehat{\mathbf{u}}
$$

in which $\widehat{\mathbf{u}}$ is the bubble displacement. It can be assumed to be

$$
\widehat{\mathbf{u}}=\left\{\begin{array}{l}
\widehat{u} \\
\widehat{v}
\end{array}\right\}=\widehat{\mathbf{N}}_{\rho} \boldsymbol{\rho}=\left[\begin{array}{ll}
\widehat{N} & 0 \\
0 & \widehat{N}
\end{array}\right]\left\{\begin{array}{l}
\rho_{1} \\
\rho_{2}
\end{array}\right\},
$$

where

$$
\widehat{N}=\left(1-\xi^{2}\right)\left(1-\eta^{2}\right)
$$

It can be considered as the shape function at the center node $\mathrm{C}$ of the element, as shown in Figure 2, which will be eliminated in the future. $\rho_{1}$ and $\rho_{2}$ in (14) are arbitrary independent parameters. Substituting (4) and (14) into (13), the element GQ12 displacement can be described in the form of

$$
\mathbf{u}=\mathbf{u}^{0}+\mathbf{u}_{\theta}+\widehat{\mathbf{u}}=\mathbf{N q} \mathbf{q}^{e}+\widehat{\mathbf{N}}_{\rho} \rho
$$

The corresponding strain fields can be expressed as

$$
\varepsilon=\mathbf{B} \mathbf{q}^{e}+\widehat{\mathbf{B}} \rho
$$

in which $\mathbf{B}$ is the same as (10), and $\widehat{\mathbf{B}}$ is the strain matrix of the bubble displacement $\widehat{\mathbf{u}}$, which can be expressed as

$$
\widehat{\mathbf{B}}=\left[\begin{array}{cc}
\frac{\partial}{\partial x^{\prime}} & 0 \\
0 & \frac{\partial}{\partial y^{\prime}} \\
\frac{\partial}{\partial y^{\prime}} & \frac{\partial}{\partial x^{\prime}}
\end{array}\right]\left[\begin{array}{cc}
\widehat{N} & 0 \\
0 & \widehat{N}
\end{array}\right]=\left[\begin{array}{cc}
\frac{\partial \widehat{N}}{\partial x^{\prime}} & 0 \\
0 & \frac{\partial \widehat{N}}{\partial y^{\prime}} \\
\frac{\partial \widehat{N}}{\partial y^{\prime}} & \frac{\partial \widehat{N}}{\partial x^{\prime}}
\end{array}\right] .
$$


According to (12) and (17), the strain energy of element GQ12M can be written as

$$
\begin{aligned}
U & =\frac{t}{2} \iint \boldsymbol{\varepsilon}^{\mathrm{T}} \mathbf{D} \boldsymbol{\varepsilon} d A \\
& =\frac{1}{2} \mathbf{q}^{e \mathrm{~T}} \mathbf{K}_{q q}^{\prime e} \mathbf{q}^{e}+\boldsymbol{\rho}^{\mathrm{T}} \mathbf{K}_{\rho q}^{\prime e} \mathbf{q}^{e}+\frac{1}{2} \boldsymbol{\rho}^{\mathrm{T}} \mathbf{K}_{\rho \rho}^{\prime e} \boldsymbol{\rho},
\end{aligned}
$$

where

$$
\begin{aligned}
\mathbf{K}_{q q}^{\prime e} & =\iint_{-1}^{1} \mathbf{B}^{\mathrm{T}} \mathbf{D} \mathbf{B}|\mathbf{J}| t d \xi d \eta, \\
\mathbf{K}_{\rho q}^{\prime e} & =\iint_{-1}^{1} \widehat{\mathbf{B}}^{\mathrm{T}} \mathbf{D} \mathbf{B}|\mathbf{J}| t d \xi d \eta, \\
\mathbf{K}_{\rho \rho}^{\prime e} & =\iint_{-1}^{1} \widehat{\mathbf{B}}^{\mathrm{T}} \mathbf{D} \widehat{\mathbf{B}}|\mathbf{J}| t d \xi d \eta
\end{aligned}
$$

in which $t$ is the thickness of the element, $|\mathbf{J}|$ is the determinant of the Jacobian matrix, and the matrices $\mathbf{B}, \mathbf{D}$, and $\widehat{\mathbf{B}}$ are given by (10), (12), and (18), respectively.

From the stationary condition $\partial U / \partial \rho=0$, the arbitrary parameters $\boldsymbol{\rho}$ can be expressed in terms of the $\mathbf{q}^{e}$ as

$$
\boldsymbol{\rho}=-\mathbf{K}_{\rho \rho}^{\prime e-1} \mathbf{K}_{\rho q}^{\prime e} \mathbf{q}^{e}
$$

Substituting (21) into (16), the shape functions of the element GQ12M can be obtained as

$$
\mathbf{N}^{*}=\mathbf{N}-\widehat{\mathbf{N}}_{\rho} \mathbf{K}_{\rho \rho}^{\prime e}{ }^{-1} \mathbf{K}_{\rho q}^{\prime e}
$$

in which $\mathbf{N}, \widehat{\mathbf{N}}_{\rho}, \mathbf{K}_{\rho \rho}^{\prime e^{-1}}$, and $\mathbf{K}_{\rho q}^{\prime e}$ are given by (5), (14), and (20), respectively.

According to the shape functions of GQ12M expressed in (22), the element stiffness matrix of element GQ12M can be written as

$$
\mathbf{K}_{\mathrm{GQ} 12 \mathrm{M}}^{\prime e}=\mathbf{K}_{q q}^{\prime e}-\mathbf{K}_{\rho q}^{\prime e^{\mathrm{T}}} \mathbf{K}_{\rho \rho}^{\prime e^{-1}} \mathbf{K}_{\rho q}^{\prime e}
$$

\section{Calculating the Stiffness Matrix of the Membrane Element under the Global Coordinate System in the Traditional Manner}

The element stiffness matrices of GQ12 and GQ12M under the local coordinate system are described in the above section, and these matrices can be numerically calculated by the Gaussian integral method. It can be seen from (9) that the GQ12 element stiffness matrix is

$$
\begin{aligned}
\mathbf{K}_{\mathrm{GQ} 12}^{\prime e} & =\iint_{-1}^{1} \mathbf{B}^{\mathrm{T}} \mathbf{D B}|\mathbf{J}| t d \xi d \eta \\
& =\sum_{i=1}^{\mathrm{NG}} \sum_{j=1}^{\mathrm{NG}} W_{i} W_{j} \mathbf{B}^{\mathrm{T}} \mathbf{D B}|\mathbf{J}| t
\end{aligned}
$$

in which $W_{i}$ and $W_{j}$ are the weight functions, and NG is the integration order. Generally, the element stiffness matrix of
TABLE 1: Roots and weight functions for $2 \times 2$ Gauss quadrature.

\begin{tabular}{lc}
\hline Roots & Weight functions $W_{i}\left(W_{j}\right)$ \\
\hline \pm 0.577350269189626 & 1.0
\end{tabular}

a 4-node quadrilateral element can be obtained using $2 \times 2$ Gauss quadrature which can achieve the exact solution of the integral equation. The roots and weight functions for $2 \times 2$ Gauss quadrature are given in Table 1 [3].

It can be seen from (11) and (24) that the shape functions with respect to the local coordinates $\partial N_{i} / \partial x^{\prime}$ and $\partial N_{i} / \partial y^{\prime}$ and the determinant of the Jacobian matrix $|\mathbf{J}|$ need to be firstly determined to calculate the GQ12 element stiffness matrices. The conventional method $[3,4]$ to compute $\partial N_{i} / \partial x^{\prime}$ and $\partial N_{i} / \partial y^{\prime}$ is to calculate the product of the inverse Jacobi matrix and the derivatives matrix of the shape functions with respect to the intrinsic coordinates, which can be expressed as

$$
\left\{\begin{array}{l}
\frac{\partial N_{i}}{\partial \xi} \\
\frac{\partial N_{i}}{\partial \eta}
\end{array}\right\}=\left[\begin{array}{ll}
\frac{\partial x^{\prime}}{\partial \xi} & \frac{\partial y^{\prime}}{\partial \xi} \\
\frac{\partial x^{\prime}}{\partial \eta} & \frac{\partial y^{\prime}}{\partial \eta}
\end{array}\right]\left\{\begin{array}{l}
\frac{\partial N_{i}}{\partial x^{\prime}} \\
\frac{\partial N_{i}}{\partial y^{\prime}}
\end{array}\right\}=\mathbf{J}\left\{\begin{array}{l}
\frac{\partial N_{i}}{\partial x^{\prime}} \\
\frac{\partial N_{i}}{\partial y^{\prime}}
\end{array}\right\}
$$

and then

$$
\begin{gathered}
\left\{\begin{array}{l}
\frac{\partial N_{i}}{\partial x^{\prime}} \\
\frac{\partial N_{i}}{\partial y^{\prime}}
\end{array}\right\}=\mathbf{J}^{-1}\left\{\begin{array}{l}
\frac{\partial N_{i}}{\partial \xi} \\
\frac{\partial N_{i}}{\partial \eta}
\end{array}\right\}, \\
|\mathbf{J}|=\left|\begin{array}{ll}
\frac{\partial x^{\prime}}{\partial \xi} & \frac{\partial y^{\prime}}{\partial \xi} \\
\frac{\partial x^{\prime}}{\partial \eta} & \frac{\partial y^{\prime}}{\partial \eta}
\end{array}\right|
\end{gathered}
$$

It is worth pointing out that $N_{i}$ in (25) and (26) is a substitution, which may be $N_{i}^{0}, N_{u \theta i}, N_{v \theta i}$, and $\widehat{N}$ in (6), (7), and (15), respectively.

Once the element local stiffness matrix for membrane element is obtained, it is necessary to transform it from the local coordinate system to the global coordinate system. So the element local coordinate system needs to be established first. In the current application of the membrane elements, a traditional manner has been adopted to establish the local Cartesian coordinate system over an element [5].

Figure 3 shows the global and local coordinate axes for the quadrilateral membrane element. The midpoints of sides 1-2, $2-3,3-4$, and $4-1$ are represented by $i, j, k$, and $l$, respectively, which can be determined by the shape functions of the four-node isoparametric element. The element local plane is defined by creating two vectors intersecting each other and passing through the midpoints of the sides 2-3 and 3-4 of the quadrilateral as shown in Figure 3. 


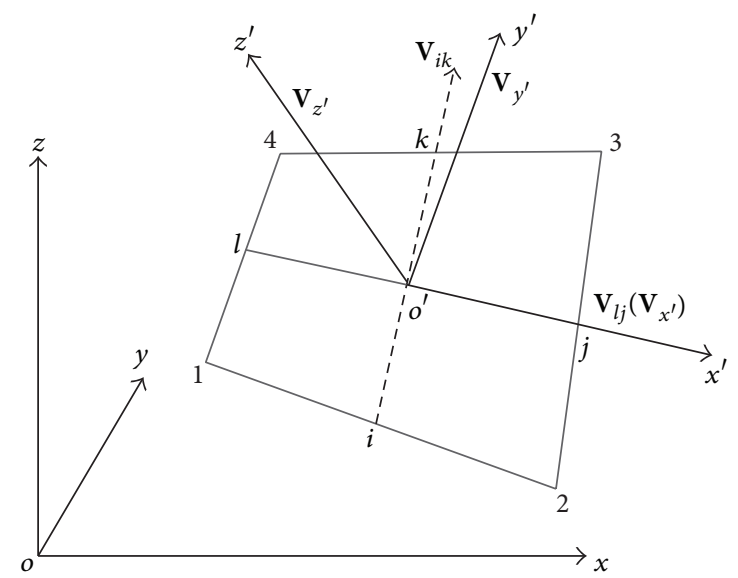

FIGURE 3: The local Cartesian coordinate system in a traditional way.

Assuming that the local $x^{\prime}$ axis of the quadrilateral is parallel to the vector passing through nodes $l$ and $j$, the vector passing through these nodes can be given by [5]

$$
\mathbf{V}_{x^{\prime}}=\mathbf{V}_{l j}=\left\{\begin{array}{c}
x_{j}-x_{l} \\
y_{j}-y_{l} \\
z_{j}-z_{l}
\end{array}\right\}=\left\{\begin{array}{l}
x_{j l} \\
y_{j l} \\
z_{j l}
\end{array}\right\},
$$

where $x_{j}, y_{j}$, and $z_{j}$ and $x_{l}, y_{l}$, and $z_{l}$ represent the global coordinates of nodes $j$ and $l$, respectively. The direction cosine $\lambda_{x^{\prime}}$ for the local $x^{\prime}$ direction can be obtained by normalizing the vector with respect to its length, which is

$$
\lambda_{x^{\prime}}=\frac{1}{l_{j l}}\left\{\begin{array}{l}
x_{j l} \\
y_{j l} \\
z_{j l}
\end{array}\right\}
$$

in which $l_{j l}=\sqrt{\left(x_{j l}\right)^{2}+\left(y_{j l}\right)^{2}+\left(z_{j l}\right)^{2}}$ is the length of the vector.

A reference vector $\mathbf{V}_{i k}$ which can define the plane of the element with the vector $\mathbf{V}_{x^{\prime}}$ is obtained by creating a vector passing through nodes $i$ and $k$ of the element as shown in Figure 3

$$
\mathbf{V}_{i k}=\left\{\begin{array}{c}
x_{k}-x_{i} \\
y_{k}-y_{i} \\
z_{k}-z_{i}
\end{array}\right\}=\left\{\begin{array}{l}
x_{k i} \\
y_{k i} \\
z_{k i}
\end{array}\right\},
$$

where $x_{k}, y_{k}$, and $z_{k}$ and $x_{i}, y_{i}$, and $z_{i}$ represent the global coordinates of nodes $k$ and $i$, respectively. The normal vector to the plane can be obtained by the vector cross product of $\mathbf{V}_{x^{\prime}}$ and $\mathbf{V}_{i k}$, which is

$$
\mathbf{V}_{z^{\prime}}=\mathbf{V}_{x^{\prime}} \times \mathbf{V}_{i k} .
$$

The direction cosine $\lambda_{z^{\prime}}$ for the local $z^{\prime}$ direction can be obtained by normalizing vector $\mathbf{V}_{z^{\prime}}$ with respect to its length. The local $y^{\prime}$ axis is obtained by the vector cross product of the vectors $\mathbf{V}_{z^{\prime}}$ and $\mathbf{V}_{x^{\prime}}$. The cross product of these two vectors will give the vector $\mathbf{V}_{y^{\prime}}$ normal to the $x^{\prime} o^{\prime} z^{\prime}$ plane, which is

$$
\mathbf{V}_{y^{\prime}}=\mathbf{V}_{z^{\prime}} \times \mathbf{V}_{x^{\prime}}
$$

The direction cosine $\lambda_{y^{\prime}}$ for the local $y^{\prime}$ direction is obtained by normalizing the vector $\mathbf{V}_{y^{\prime}}$ with respect to its length, with reference to (28).

So the $3 \times 3$ transformation matrix $\mathbf{L}$ for the transformation of coordinates from the local to the global axis can be expressed as

$$
\mathbf{L}=\left[\begin{array}{lll}
\left(\lambda_{x^{\prime}}\right)_{3 \times 1} & \left(\lambda_{y^{\prime}}\right)_{3 \times 1} & \left(\lambda_{z^{\prime}}\right)_{3 \times 1}
\end{array}\right]^{\mathrm{T}}
$$

in which $\lambda_{x^{\prime}}, \lambda_{y^{\prime}}$, and $\lambda_{z^{\prime}}$ are the direction cosines for local $x^{\prime}$, $y^{\prime}$, and $z^{\prime}$ directions. The $12 \times 12$ transformation matrix $\mathbf{R}$ for the 4-node quadrilateral element stiffness from the local to the global coordinate systems can be represented by $\mathbf{L}$, which is

$$
\mathbf{R}=\left[\begin{array}{llll}
\mathbf{L} & & & \\
& \mathbf{L} & & \\
& & \mathbf{L} & \\
& & & \mathbf{L}
\end{array}\right]
$$

To calculate the structure stiffness matrix, the element stiffness matrix must be transformed to the global coordinate system. The transformation of the GQ12 element stiffness matrix from the local to the global coordinate system is given by $[3,4]$

$$
\begin{aligned}
\mathbf{K}_{\mathrm{GQ} 12}^{e} & =\mathbf{R}^{\mathrm{T}} \mathbf{K}_{\mathrm{GQ} 12}^{\prime e} \mathbf{R} \\
& =\mathbf{R}^{\mathrm{T}}\left(\sum_{i=1}^{\mathrm{NG}} \sum_{j=1}^{\mathrm{NG}} W_{i} W_{j} \mathbf{B}^{\mathrm{T}} \mathbf{D B}|\mathbf{J}| t\right) \mathbf{R} .
\end{aligned}
$$

Similarly, from (20) and (23), the GQ12M element stiffness matrix under the global coordinate system can be described as

$$
\begin{aligned}
\mathbf{K}_{\mathrm{GQ} 12 \mathrm{M}}^{e} & =\mathbf{R}^{\mathrm{T}} \mathbf{K}_{\mathrm{GQ} 12 \mathrm{M}}^{\prime e} \mathbf{R} \\
& =\mathbf{R}^{\mathrm{T}} \mathbf{K}_{q q}^{\prime e} \mathbf{R}-\left(\mathbf{K}_{\rho q}^{\prime e} \mathbf{R}\right)^{\mathrm{T}}\left(\mathbf{K}_{\rho \rho}^{\prime e}\right)^{-1}\left(\mathbf{K}_{\rho q}^{\prime e} \mathbf{R}\right)
\end{aligned}
$$

in which

$$
\begin{aligned}
\mathbf{R}^{\mathrm{T}} \mathbf{K}_{q q}^{\prime e} \mathbf{R} & =\mathbf{R}^{\mathrm{T}}\left(\sum_{i=1}^{\mathrm{NG}} \sum_{j=1}^{\mathrm{NG}} W_{i} W_{j} \mathbf{B}^{\mathrm{T}} \mathbf{D B}|\mathbf{J}| t\right) \mathbf{R}, \\
\mathbf{K}_{\rho q}^{\prime e} \mathbf{R} & =\left(\sum_{i=1}^{\mathrm{NG}} \sum_{j=1}^{\mathrm{NG}} W_{i} W_{j} \widehat{\mathbf{B}}^{\mathrm{T}} \mathbf{D B}|\mathbf{J}| t\right) \mathbf{R} .
\end{aligned}
$$

It can be seen from the above equations that the key task of computing the membrane stiffness matrix is to accurately evaluate the derivatives of the shape functions with respect to the local coordinates $\partial N_{i} / \partial x^{\prime}$ and $\partial N_{i} / \partial y^{\prime}$, the determinant 
of the Jacobian matrix $|\mathbf{J}|$, and transformation matrix $\mathbf{R}$. The membrane element in the three-dimensional space may be not regular and coplanar. Thus, the calculation accuracy of the traditional way to establish the element local Cartesian coordinate system is heavily dependent on the element regularity.

In order to improve the performance of the membrane elements GQ12 and GQ12M, a new method to establish the element local Cartesian coordinate system is proposed. And the corresponding techniques to calculate the derivatives of the shape functions with respect to the local coordinates, the transformation matrix from the local to the global coordinate systems, and the determinant of the Jacobian matrix are also provided in this paper $[26,27]$.

\section{A New Method to Calculate the Stiffness Matrix of the Membrane Element in the Global Coordinate System}

4.1. Establishing the Local Coordinate System. In an effort to avoid confusion and awkward phrasing, the signs $\left(x_{1}, x_{2}, x_{3}\right)$, $\left(x_{1}^{\prime}, x_{2}^{\prime}, x_{3}^{\prime}\right)$, and $(\xi, \eta)$ are used to denote the symbols of the global Cartesian coordinate system, the local Cartesian coordinate system, and the intrinsic coordinates system, respectively.

In order to improve the calculation accuracy, a new way [26] to establish the local Cartesian coordinate system is suggested in this paper. As shown in Figure 4, the origin of the local Cartesian coordinate system is set to the origin of the intrinsic coordinate system. Firstly, the vectors $\mathbf{r}_{\xi}$ and $\mathbf{r}_{\eta}$ from the origin need to be built along the tangent directions of the axes $\xi$ and $\eta$, respectively, as illustrated in Figure 5. The direction of the vector $\mathbf{r}_{\xi}$ is considered as the local $x_{1}^{\prime}$ direction. Then the vectors $\mathbf{r}_{\xi}$ and $\mathbf{r}_{\eta}$ define the plane which is tangential to the element surface. The normal vector $\mathbf{n}$ to this plane can be obtained by the vector cross product of $\mathbf{r}_{\xi}$ and $\mathbf{r}_{\eta}$, which is the local $x_{3}^{\prime}$ direction. At last, the local $x_{2}^{\prime}$ axis is obtained by the vector cross product of the vector in the local $x_{1}^{\prime}$ direction and vector in local $x_{3}^{\prime}$ direction. Now the local Cartesian coordinate system in which the axes $x_{1}^{\prime}$ and $x_{2}^{\prime}$ are tangential to the surface and $x_{3}^{\prime}$ is directed in the normal direction (shown in Figure 4) is established successfully.

However, when the curvature of element surface is large, the numerical results are still not very accurate in this local Cartesian coordinate system. Therefore, in order to further enhance the precision of the calculation, the origin of the local Cartesian coordinate system can be set at the Gauss points. This idea came from the traction recovery method [28-31] in Boundary Element Method (BEM) which establishes the local Cartesian coordinate systems at each node of the element in order to improve the calculation accuracy. In finite element method (FEM), the numerical integration is performed on Gauss points, so the local Cartesian coordinate systems can be established at Gauss points. Take the $2 \times$ 2 Gauss point integration as an example, as indicated in Figure 6; the local Cartesian coordinate systems with the

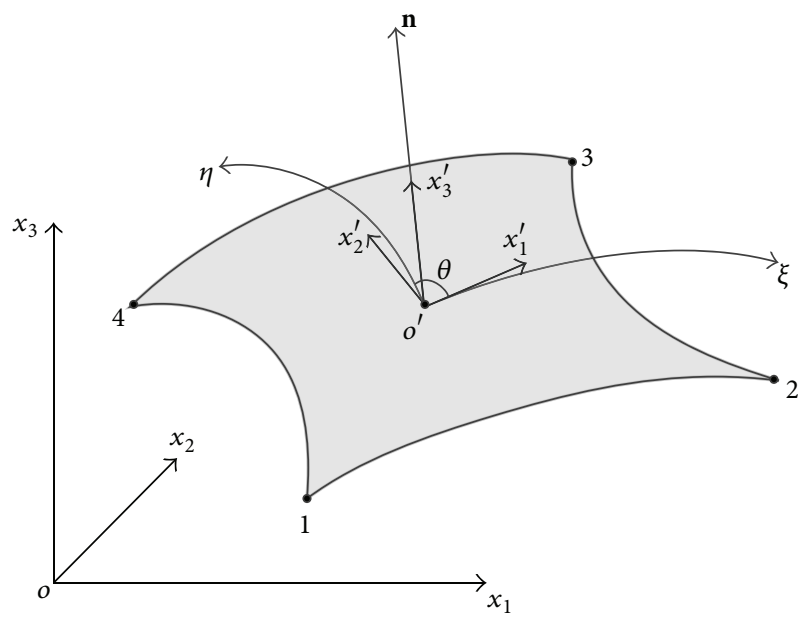

FIGURE 4: Local orthogonal set of axes over a surface element in the new way.

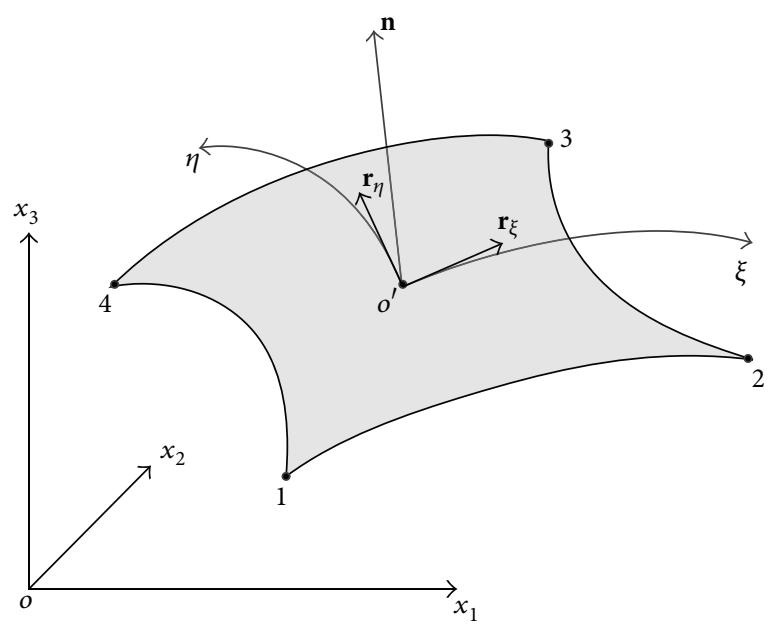

Figure 5: The vectors $\mathbf{r}_{\xi}$ and $\mathbf{r}_{\eta}$ in the local tangent plane to the surface.

origins at the four Gauss points can be established in a similar way as shown in Figure 4.

It is reasonable to establish the local Cartesian coordinate systems at Gauss points since the numerical integration is performed on them. In this case, the calculation accuracy can be improved, because the local Cartesian coordinate systems which are established on the tangent plane to the element surface at Gauss points can adapt to the curved element surface better. In order to better describe this problem, an example shown in Figure 7 which is a curved element surface is introduced to illustrate the performance of this new local Cartesian coordinate systems established at Gauss points. Figure 8 describes the local Cartesian coordinate system established in the traditional method at the curved element surface. It can be observed that the element local plane has a large difference with the curved element surface. By comparison, the element local planes defined by the local Cartesian coordinate systems established at $2 \times 2$ Gauss points are more accordant with the curved element surface, 


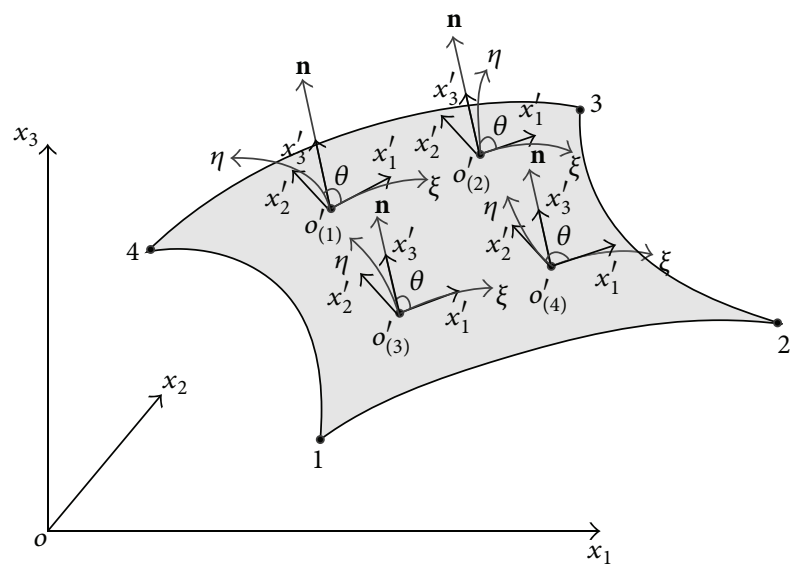

FIGURE 6: Local Cartesian coordinate systems with the origins at the Gauss points.

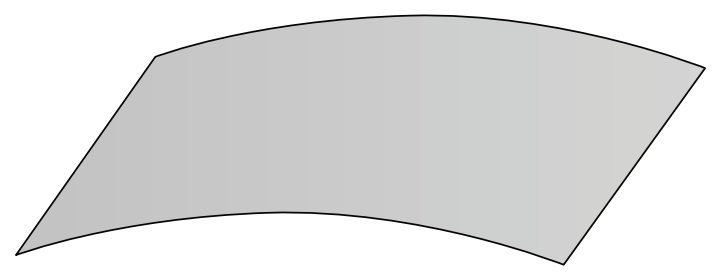

FIGURE 7: A curved element surface.

as illustrated in Figure 9. In essence, Gaussian integration is the summation of the numerical results at Gauss points, so it is reasonable to believe that the calculation accuracy of the numerical integration can be improved by establishing the local Cartesian coordinate system at each Gauss point.

As shown in Figure 9, the local Cartesian coordinate systems are different since they are established in different tangent planes to the curved element surface. So the direction cosines $\lambda_{x^{\prime}}, \lambda_{y^{\prime}}$, and $\lambda_{z^{\prime}}$ of the local coordinate system established at different Gauss point with respect to the global coordinate system are different with each other. The detailed formulas and computation steps of $\boldsymbol{\lambda}_{x^{\prime}}, \boldsymbol{\lambda}_{y^{\prime}}$, and $\boldsymbol{\lambda}_{z^{\prime}}$ will be given in the following sections. According to (31) and (32), it can be seen that the transformation matrix $\mathbf{R}$ for the transformation of element stiffness matrix from the local to the global coordinate systems may be different. So the element stiffness matrices of GQ12 and GQ12M under the global coordinate system expressed as (33), (35), and (36) can be modified to be

$$
\begin{aligned}
\mathbf{K}_{\mathrm{GQ} 12}^{e} & =\sum_{i=1}^{\mathrm{NG}} \sum_{j=1}^{\mathrm{NG}} W_{i} W_{j}(\mathbf{B R})^{\mathrm{T}} \mathbf{D}(\mathbf{B R})|\mathbf{J}| t, \\
\mathbf{R}^{\mathrm{T}} \mathbf{K}_{q q}^{\prime e} \mathbf{R} & =\sum_{i=1}^{\mathrm{NG}} \sum_{j=1}^{\mathrm{NG}} W_{i} W_{j}(\mathbf{B R})^{\mathrm{T}} \mathbf{D}(\mathbf{B R})|\mathbf{J}| t, \\
\mathbf{K}_{\rho q}^{\prime e} \mathbf{R} & =\sum_{i=1}^{\mathrm{NG}} \sum_{j=1}^{\mathrm{NG}} W_{i} W_{j} \widehat{\mathbf{B}}^{\mathrm{T}} \mathbf{D B R}|\mathbf{J}| t .
\end{aligned}
$$

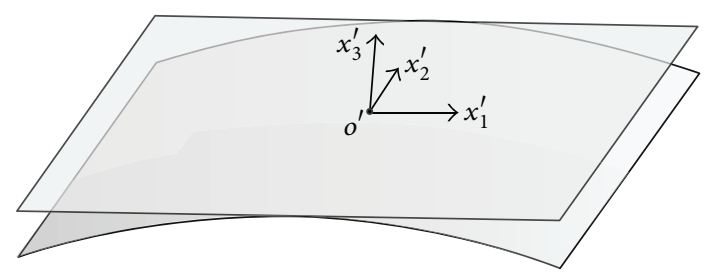

Figure 8: The local Cartesian coordinate system established in the traditional method and the element local plane at the curved element surface.

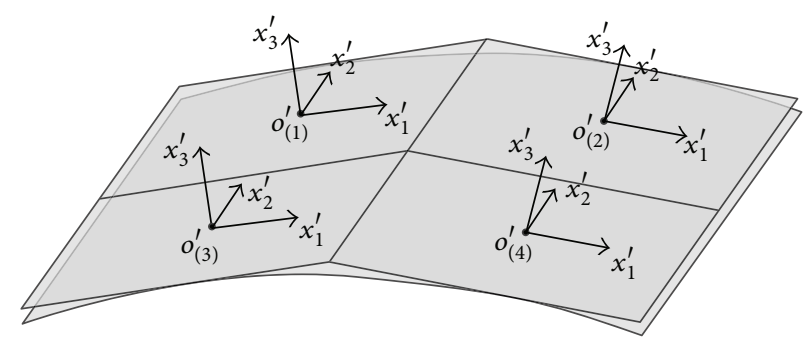

FIgURE 9: The local Cartesian coordinate systems established in the new method and the element local planes at the curved element surface.

4.2. Derivatives of Shape Functions with respect to Local Coordinates. According to the derivation rule for compound function, the derivatives of the shape functions with respect to the local coordinates can be written as

$$
\left\{\begin{array}{l}
\frac{\partial N_{i}}{\partial x_{1}^{\prime}} \\
\frac{\partial N_{i}}{\partial x_{2}^{\prime}}
\end{array}\right\}=\left[\begin{array}{ll}
\frac{\partial \xi}{\partial x_{1}^{\prime}} & \frac{\partial \eta}{\partial x_{1}^{\prime}} \\
\frac{\partial \xi}{\partial x_{2}^{\prime}} & \frac{\partial \eta}{\partial x_{2}^{\prime}}
\end{array}\right]\left\{\begin{array}{l}
\frac{\partial N_{i}}{\partial \xi} \\
\frac{\partial N_{i}}{\partial \eta}
\end{array}\right\}, \quad(i=1,2,3,4)
$$

in which $\partial \xi / \partial x_{j}^{\prime}$ and $\partial \eta / \partial x_{j}^{\prime}(j=1,2)$ are the derivatives of the intrinsic coordinates with respect to the local coordinates, which can be evaluated using the method proposed by Lachat $[26,32-34]$; that is,

$$
\begin{aligned}
\frac{\partial \xi}{\partial x_{1}^{\prime}} & =\frac{1}{\left|m_{1}\right|}, \\
\frac{\partial \xi}{\partial x_{2}^{\prime}} & =\frac{-\cos \theta}{\left|m_{1}\right| \sin \theta}, \\
\frac{\partial \eta}{\partial x_{1}^{\prime}} & =0, \\
\frac{\partial \eta}{\partial x_{2}^{\prime}} & =\frac{1}{\left|m_{2}\right| \sin \theta},
\end{aligned}
$$


where $\theta$ is the angle between $x_{1}^{\prime}$ axis and $\eta$ axis shown in Figure 4, and

$$
\begin{aligned}
& \left|m_{1}\right|=\sqrt{\left(\frac{\partial x_{1}}{\partial \xi}\right)^{2}+\left(\frac{\partial x_{2}}{\partial \xi}\right)^{2}+\left(\frac{\partial x_{3}}{\partial \xi}\right)^{2}}, \\
& \left|m_{2}\right|=\sqrt{\left(\frac{\partial x_{1}}{\partial \eta}\right)^{2}+\left(\frac{\partial x_{2}}{\partial \eta}\right)^{2}+\left(\frac{\partial x_{3}}{\partial \eta}\right)^{2}}, \\
& \cos \theta=\frac{1}{\left|m_{1}\right|\left|m_{2}\right|} \frac{\partial x_{i}}{\partial \xi} \frac{\partial x_{i}}{\partial \eta}, \quad(i=1,2,3)
\end{aligned}
$$

in which $x_{i}(i=1,2,3)$ is the global coordinates of the element nodes, and $\xi, \eta$ are the intrinsic coordinates.

In (39), $N_{i}$ is a substitution, which may be $N_{i}^{0}, N_{u \theta i}$, $N_{v \theta i}$, and $\widehat{N}$ in (6), (7), and (15), respectively. So separately substituting (6), (7), and (15) into (39) gives

$$
\begin{aligned}
& \left\{\begin{array}{l}
\frac{\partial N_{i}^{0}}{\partial x^{\prime}} \\
\frac{\partial N_{i}^{0}}{\partial y^{\prime}}
\end{array}\right\}=\left\{\begin{array}{l}
\frac{\partial N_{i}^{0}}{\partial x_{1}^{\prime}} \\
\frac{\partial N_{i}^{0}}{\partial x_{2}^{\prime}}
\end{array}\right\}=\left[\begin{array}{cc}
\frac{\partial \xi}{\partial x_{1}^{\prime}} & \frac{\partial \eta}{\partial x_{1}^{\prime}} \\
\frac{\partial \xi}{\partial x_{2}^{\prime}} & \frac{\partial \eta}{\partial x_{2}^{\prime}}
\end{array}\right]\left\{\begin{array}{c}
\frac{\partial N_{i}^{0}}{\partial \xi} \\
\frac{\partial N_{i}^{0}}{\partial \eta}
\end{array}\right\}, \\
& (i=1,2,3,4), \\
& \left\{\begin{array}{c}
\frac{\partial N_{u \theta i}}{\partial x^{\prime}} \\
\frac{\partial N_{u \theta i}}{\partial y^{\prime}}
\end{array}\right\}=\left\{\begin{array}{c}
\frac{\partial N_{u \theta i}}{\partial x_{1}^{\prime}} \\
\frac{\partial N_{u \theta i}}{\partial x_{2}^{\prime}}
\end{array}\right\}=\left[\begin{array}{cc}
\frac{\partial \xi}{\partial x_{1}^{\prime}} & \frac{\partial \eta}{\partial x_{1}^{\prime}} \\
\frac{\partial \xi}{\partial x_{2}^{\prime}} & \frac{\partial \eta}{\partial x_{2}^{\prime}}
\end{array}\right]\left\{\begin{array}{c}
\frac{\partial N_{u \theta i}}{\partial \xi} \\
\frac{\partial N_{u \theta i}}{\partial \eta}
\end{array}\right\}, \\
& (i=1,2,3,4), \\
& \left\{\begin{array}{c}
\frac{\partial N_{v \theta i}}{\partial x^{\prime}} \\
\frac{\partial N_{v \theta i}}{\partial y^{\prime}}
\end{array}\right\}=\left\{\begin{array}{c}
\frac{\partial N_{v \theta i}}{\partial x_{1}^{\prime}} \\
\frac{\partial N_{v \theta i}}{\partial x_{2}^{\prime}}
\end{array}\right\}=\left[\begin{array}{ll}
\frac{\partial \xi}{\partial x_{1}^{\prime}} & \frac{\partial \eta}{\partial x_{1}^{\prime}} \\
\frac{\partial \xi}{\partial x_{2}^{\prime}} & \frac{\partial \eta}{\partial x_{2}^{\prime}}
\end{array}\right]\left\{\begin{array}{c}
\frac{\partial N_{v \theta i}}{\partial \xi} \\
\frac{\partial N_{v \theta i}}{\partial \eta}
\end{array}\right\}, \\
& (i=1,2,3,4), \\
& \left\{\begin{array}{l}
\frac{\partial \widehat{N}}{\partial x^{\prime}} \\
\frac{\partial \widehat{N}}{\partial y^{\prime}}
\end{array}\right\}=\left\{\begin{array}{l}
\frac{\partial \widehat{N}}{\partial x_{1}^{\prime}} \\
\frac{\partial \widehat{N}}{\partial x_{2}^{\prime}}
\end{array}\right\}=\left[\begin{array}{ll}
\frac{\partial \xi}{\partial x_{1}^{\prime}} & \frac{\partial \eta}{\partial x_{1}^{\prime}} \\
\frac{\partial \xi}{\partial x_{2}^{\prime}} & \frac{\partial \eta}{\partial x_{2}^{\prime}}
\end{array}\right]\left\{\begin{array}{l}
\frac{\partial \widehat{N}}{\partial \xi} \\
\frac{\partial \widehat{N}}{\partial \eta}
\end{array}\right\} .
\end{aligned}
$$

The derivatives of the shape functions with respect to the local coordinates can be obtained by substituting (40) into the above equations. Then the strain matrices $\mathbf{B}$ and $\widehat{\mathbf{B}}$ can be obtained by substituting the above equations into (10) and (18), respectively.

4.3. Determination of the Jacobian $|\mathbf{J}|$. For the convenience of description, the local Cartesian coordinate system involved in this section is based on the way as shown in Figure 4. For the local Cartesian coordinate systems with the origins at the Gauss points, the approach to determine $|\mathbf{J}|$ is similar.

Referring to Figure 10, the Jacobian is equal to the magnitude of the vector cross product of the vectors $\mathbf{r}_{\xi}$ and $\mathbf{r}_{\eta}$, which

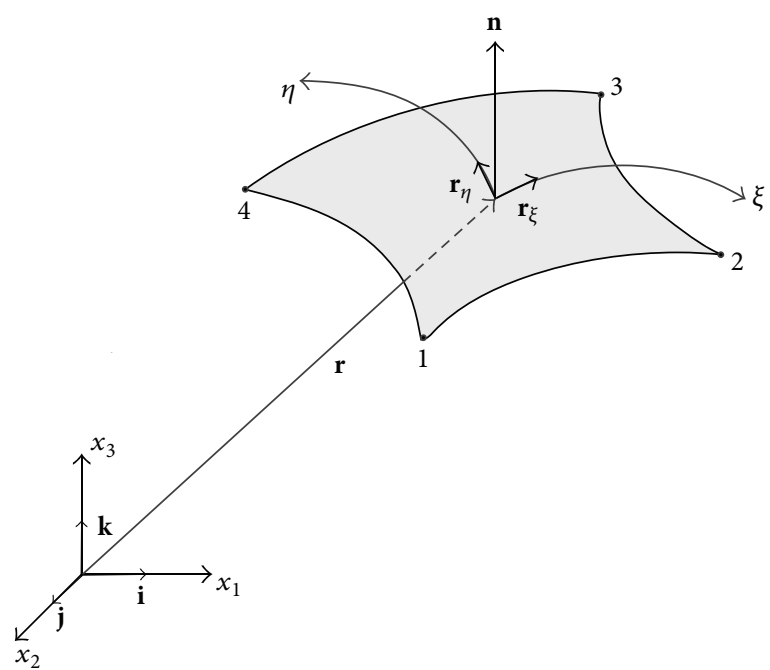

Figure 10: Normal vector to a surface element.

are located in the local tangent plane to the surface as can also be seen in Figure 5. The Jacobian of the transformation from the global three-dimensional coordinate system to the twodimensional intrinsic coordinate system of the surface patch is introduced in the form of [26]

$$
|\boldsymbol{J}|^{\prime}=\left|\mathbf{n}^{*}\right|=\left|\mathbf{r}_{\xi} \times \mathbf{r}_{\eta}\right|,
$$

where

$$
\begin{aligned}
\mathbf{r}_{\xi}= & \frac{\partial x_{1}(\xi, \eta)}{\partial \xi} \mathbf{i}+\frac{\partial x_{2}(\xi, \eta)}{\partial \xi} \mathbf{j}+\frac{\partial x_{3}(\xi, \eta)}{\partial \xi} \mathbf{k}, \\
\mathbf{r}_{\eta}= & \frac{\partial x_{1}(\xi, \eta)}{\partial \eta} \mathbf{i}+\frac{\partial x_{2}(\xi, \eta)}{\partial \eta} \mathbf{j}+\frac{\partial x_{3}(\xi, \eta)}{\partial \eta} \mathbf{k}, \\
\mathbf{n}^{*} & =\mathbf{r}_{\xi} \times \mathbf{r}_{\eta}=\left(\frac{\partial x_{2}(\xi, \eta)}{\partial \xi} \cdot \frac{\partial x_{3}(\xi, \eta)}{\partial \eta}-\frac{\partial x_{3}(\xi, \eta)}{\partial \xi}\right. \\
& \left.\cdot \frac{\partial x_{2}(\xi, \eta)}{\partial \eta}\right) \mathbf{i}+\left(\frac{\partial x_{3}(\xi, \eta)}{\partial \xi} \cdot \frac{\partial x_{1}(\xi, \eta)}{\partial \eta}\right. \\
& \left.-\frac{\partial x_{1}(\xi, \eta)}{\partial \xi} \cdot \frac{\partial x_{3}(\xi, \eta)}{\partial \eta}\right) \mathbf{j}+\left(\frac{\partial x_{1}(\xi, \eta)}{\partial \xi}\right. \\
& \left.\cdot \frac{\partial x_{2}(\xi, \eta)}{\partial \eta}-\frac{\partial x_{2}(\xi, \eta)}{\partial \xi} \cdot \frac{\partial x_{1}(\xi, \eta)}{\partial \eta}\right) \mathbf{k},
\end{aligned}
$$

in which $\mathbf{i}, \mathbf{j}$, and $\mathbf{k}$ are the orthogonal unit basis vectors of the global coordinate axes and $\mathbf{n}^{*}$ is normal to the surface. So the unit normal vector may be obtained from

$$
\mathbf{n}=\frac{\mathbf{n}^{*}}{\left|\mathbf{n}^{*}\right|}=n_{1} \mathbf{i}+n_{2} \mathbf{j}+n_{3} \mathbf{k},
$$

in which $n_{1}, n_{2}$, and $n_{3}$ are the magnitude of the components of the unit normal vector in the global coordinate axes.

The Jacobian of the transformation from the local orthogonal coordinate system to the intrinsic coordinate system is the same as that from the global orthogonal to the intrinsic coordinate systems. So the value of $|\mathbf{J}|$ in (38) can be computed using (44). 


\section{The Matrix Algorithm for the Membrane Element Stiffness Transformation from the Local to the Global Coordinate Systems}

As described in Figure 4, the direction of local $x_{1}^{\prime}$ axis is the tangent direction of the axis $\xi$ at the origin, which is the direction of the vector $\mathbf{r}_{\xi}$. So the direction cosine $\lambda_{x_{1}^{\prime}}$ for the local $x_{1}^{\prime}$ direction is obtained by normalizing the vector $\mathbf{r}_{\xi}$ with respect to its length. According to (41) and (45), the direction cosine $\lambda_{x_{1}^{\prime}}$ can be written as [26]

$$
\lambda_{x^{\prime}}=\lambda_{x_{1}^{\prime}}=\frac{1}{\left|m_{1}\right|}\left\{\begin{array}{l}
\frac{\partial x_{1}}{\partial \xi} \\
\frac{\partial x_{2}}{\partial \xi} \\
\frac{\partial x_{3}}{\partial \xi}
\end{array}\right\},
$$

in which $x_{i}(i=1,2,3)$ is the global coordinates of the element nodes and $\left|m_{1}\right|$ is the mold of the vector $\mathbf{r}_{\xi}$ expressed in (41). The local $x_{3}^{\prime}$ direction is the direction of the normal vector $\mathbf{n}$ to the element surface which can be obtained from (47). So the direction cosine $\boldsymbol{\lambda}_{x_{3}^{\prime}}$ can be written as

$$
\lambda_{z^{\prime}}=\lambda_{x_{3}^{\prime}}=\left\{\begin{array}{l}
n_{1} \\
n_{2} \\
n_{3}
\end{array}\right\}
$$

in which $n_{1}, n_{2}$, and $n_{3}$ are given by (47). Because the local $x_{2}^{\prime}$ axis can be obtained by the vector cross product of the vector in the local $x_{1}^{\prime}$ direction and vector in local $x_{3}^{\prime}$ direction, the direction cosine $\lambda_{x_{2}^{\prime}}$ can be written as

$$
\lambda_{y^{\prime}}=\lambda_{x_{2}^{\prime}}=\lambda_{z^{\prime}} \times \lambda_{x^{\prime}}
$$

So the $3 \times 3$ transformation matrix $\mathbf{L}$ for the transformation of coordinates from the local to the global axis can be obtained from (32), which can be expressed in terms of $L_{i j}(i, j=1,2,3)$ as [26]

$$
\begin{aligned}
L_{1 j} & =\frac{1}{\left|m_{1}\right|} \frac{\partial x_{j}}{\partial \xi}, \quad(j=1,2,3), \\
L_{21} & =n_{2} L_{13}-n_{3} L_{12}, \\
L_{22} & =n_{3} L_{11}-n_{1} L_{13}, \\
L_{23} & =n_{1} L_{12}-n_{2} L_{11}, \\
L_{3 j} & =n_{j}, \quad(j=1,2,3) .
\end{aligned}
$$

The $12 \times 12$ transformation matrix $\mathbf{R}$ for the 4-node quadrilateral element stiffness from the local to the global coordinate systems can be obtained from (33). It is worth mentioning that the matrix $\mathbf{R}$ is just used for the membrane element stiffness transformation from the local to the global coordinate systems. For the 4-node quadrilateral shell
TABLE 2: The vertical deflection results of Cook's problem.

Vertical deflection at $\mathrm{C}(\mathrm{m})$

\begin{tabular}{lccc} 
Element & \multicolumn{3}{c}{$(2 \times 2)$ mesh } \\
& Deflection & Deflection & Deflection \\
\hline Q4 [4] & 11.80 & 18.29 & 22.08 \\
Allman [15] & 20.27 & 22.78 & 23.56 \\
GQ12 [25] & 20.89 & 23.06 & 23.67 \\
GQ12 (present method) & 21.27 & 23.07 & 23.67 \\
GQ12M [25] & 21.69 & 23.30 & 23.74 \\
GQ12M (present method) & 22.47 & 23.43 & 23.78 \\
Comparison solution [35] & & 23.96 & \\
\hline
\end{tabular}

element which has six degrees of freedom in each node, the $24 \times 24$ transformation matrix $\mathbf{T}$ for the transformation of shell element stiffness from the local to the global coordinate systems is written as [4]

$$
\mathbf{T}=\left[\begin{array}{ll}
\mathbf{R} & \\
& \mathbf{R}
\end{array}\right] .
$$

\section{Numerical Examples}

In order to examine the performance of the new method for GQ12 and GQ12M described in this paper, four numerical examples are proposed in this section. And the computational results are compared with those calculated by the traditional method based on the elements GQ12 and GQ12M.

6.1. Example 1: Cook's Problem. The problem defined in Figure 11 was proposed by Cook [36] as a test case of plane stress elements, which is a standard example for accuracy testing of plane stress problem. Young's modulus of the element is $E=1 \mathrm{~Pa}$, and Poisson's ratio is $v=1 / 3$. The structure is meshed with $2 \times 2,4 \times 4$, and $8 \times 8$ elements. Here the new methods based on the elements GQ12 and GQ12M proposed in this paper are utilized to solve this problem. The best known answers taken from [35] are used for comparison, since there is no analytical solution available for this problem. The results of the vertical deflection at $\mathrm{C}$, the maximum stress at $\mathrm{A}$, and the minimum stress at $\mathrm{B}$ on different meshes of the structure are given in Tables 2 and 3. The results demonstrate that the new method for the elements GQ12 and GQ12M have the desirable numerical accuracy, both for the displacement and for the stress.

6.2. Example 2: MacNeal Beam. In order to examine the membrane locking phenomena, a typical test example $[37,38]$ defined in Figure 12 was proposed by MacNeal. The thickness of the membrane elements is $t=0.1 \mathrm{~m}$, Young's modulus is $E=10^{7} \mathrm{~Pa}$, and Poisson's ratio is $v=0.3$. The beam end displacements obtained with several elements under the unit shear load at the free end are compared with the theoretical values taken from [38]. The results under different meshes in Table 4 demonstrate that GQ12 and GQ12M with the new method described in this paper can delete membrane locking and have the same accuracy as the traditional method. 
TABLE 3: The stress results of Cook's problem.

\begin{tabular}{|c|c|c|c|c|c|c|}
\hline \multirow{3}{*}{ Element } & \multicolumn{3}{|c|}{ Maximum stress at $\mathrm{A}(\mathrm{Pa})$} & \multicolumn{3}{|c|}{ Minimum stress at $\mathrm{B}(\mathrm{Pa})$} \\
\hline & \multicolumn{2}{|c|}{$(2 \times 2)$ mesh } & \multicolumn{2}{|c|}{$(4 \times 4)$ mesh } & \multicolumn{2}{|c|}{$(8 \times 8)$ mesh } \\
\hline & $\sigma_{\mathrm{A}}$ & $\sigma_{\mathrm{B}}$ & $\sigma_{\mathrm{A}}$ & $\sigma_{\mathrm{B}}$ & $\sigma_{\mathrm{A}}$ & $\sigma_{\mathrm{B}}$ \\
\hline Q4 [4] & 0.1217 & -0.0957 & 0.1873 & -0.1524 & 0.2242 & -0.1869 \\
\hline Allman [15] & 0.1825 & -0.1716 & 0.2261 & -0.1921 & 0.2340 & -0.2004 \\
\hline GQ12 [25] & 0.1802 & -0.1784 & 0.2209 & -0.1950 & 0.2315 & -0.2007 \\
\hline GQ12 (present method) & 0.1841 & -0.1810 & 0.2214 & -0.1948 & 0.2315 & -0.2008 \\
\hline GQ12M [25] & 0.1861 & -0.2088 & 0.2232 & -0.2017 & 0.2305 & -0.2017 \\
\hline GQ12M (present method) & 0.2070 & -0.2142 & 0.2333 & -0.2037 & 0.2361 & -0.2027 \\
\hline Comparison solution [35] & & $\sigma_{\mathrm{A}}=0.2362$ & & & $=-0.202$ & \\
\hline
\end{tabular}

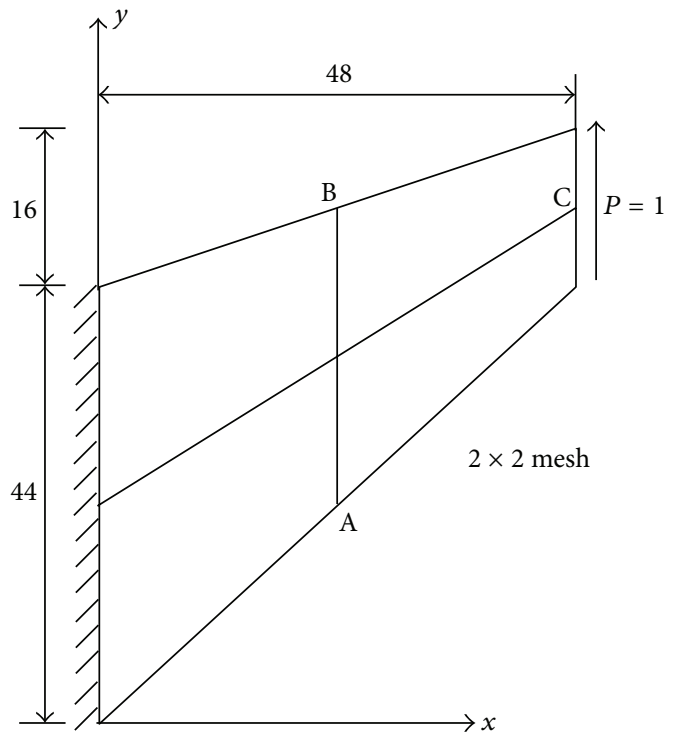

FIGURE 11: Cook's problem.

TABLE 4: The results of the MacNeal beam under different meshes.

\begin{tabular}{lccc}
\hline \multirow{2}{*}{ Element } & \multicolumn{3}{c}{ The beam end deflection (m) } \\
& Mesh (a) & Mesh (b) & Mesh (c) \\
\hline Q4 [4] & -0.0904 & -0.0710 & -0.0800 \\
GQ12 [25] & -0.0904 & -0.0767 & -0.0860 \\
GQ12 (present method) & -0.0977 & -0.0871 & -0.0944 \\
GQ12M [25] & -0.0977 & -0.0886 & -0.0923 \\
GQ12M (present method) & -0.1073 & -0.0980 & -0.1050 \\
Theoretical value [38] & & -0.1081 & \\
\hline
\end{tabular}

6.3. Example 3: Uniform Stretching (Patch Test) and Bending of a Plate. This test example [25] is a rectangular plate under two loading cases, which are the uniform stretching under Load 1 and the pure bending under Load 2, respectively. The uniform stretching case is the patch test problem under constant strain. Because of the symmetry of the model and loads, only a quarter of the plate with the irregular mesh shown in Figure 13 is considered. Young's modulus of the element is $E=1 \mathrm{~Pa}$, and Poisson's ratio is $v=1 / 3$. Table 5
TABle 5: The displacement at corner A under two loading cases.

\begin{tabular}{lcc}
\hline Element & Load 1 & Load 2 \\
& $U_{\mathrm{A}}(\mathrm{m})$ & $V_{\mathrm{A}}(\mathrm{m})$ \\
\hline Q4 [4] & 6.00 & -17.00 \\
GQ12 [25] & 6.00 & -17.72 \\
GQ12 (present method) & 6.00 & -17.75 \\
GQ12M [25] & 6.00 & -17.77 \\
GQ12M (present method) & 6.00 & -17.82 \\
Exact [39] & 6.00 & -18.00 \\
\hline
\end{tabular}

shows the results of displacement at corner A which are obtained with different elements under these two loading cases. It demonstrates that the elements GQ12 and GQ12M with present method in this paper pass the patch test for a general quadrilateral mesh and achieve more accurate results.

6.4. Example 4: Linear Analysis of a Hemispherical Shell. The aim of this test example [40] is to examine the performance of GQ12 and GQ12M elements with the present method in this paper as a constituent part of the flat shell element to compute a curved shell structure. As known, the flat shell elements are formed by superimposing the stiffness of membrane and plate bending elements. In this section, the plate bending element is based on the Mindlin theory which includes transverse shear deformations, and the membrane element may be selected from GQ12 and GQ12M with present method in this paper, respectively.

Figure 14 shows a hemispherical shell structure with an 18-degree hole at the top [40]. The radius of the shell is $10 \mathrm{~m}$, thickness is $0.04 \mathrm{~m}$, Young's modulus is $6.825 \times 10^{7} \mathrm{~Pa}$, and Poisson's ratio is 0.3 . The top and bottom circumferential edges of the hemisphere are free and the shell is subjected to two radial unit point loads. Only a quarter of the hemispherical shell with the meshes shown in Figure 15 is separated out for research due to the geometric symmetry. The radial displacement at point $\mathrm{A}$ from different meshes is compared with the theoretical solution $u_{\mathrm{A}}=0.094$ [38] in Table 6. It can be seen that the shell elements based on the membrane elements with present method in this paper can achieve more accurate results than those based on a traditional manner. 


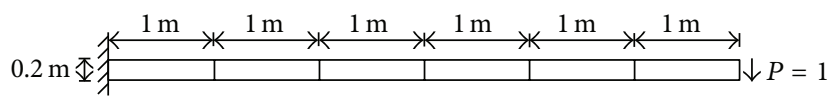

(a) Rectangular mesh

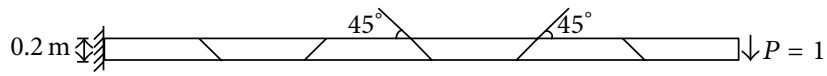

(b) Trapezoid mesh

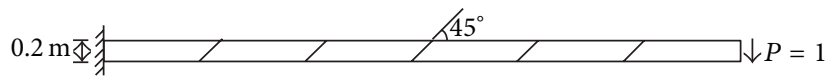

(c) Rhomboid mesh

Figure 12: MacNeal beam.

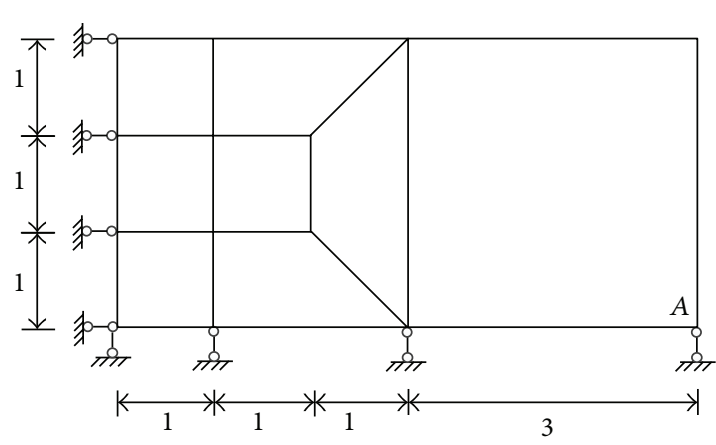

(a) Uniform stretching problem (patch test problem)

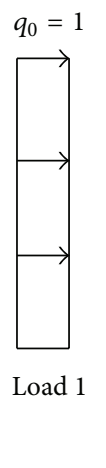

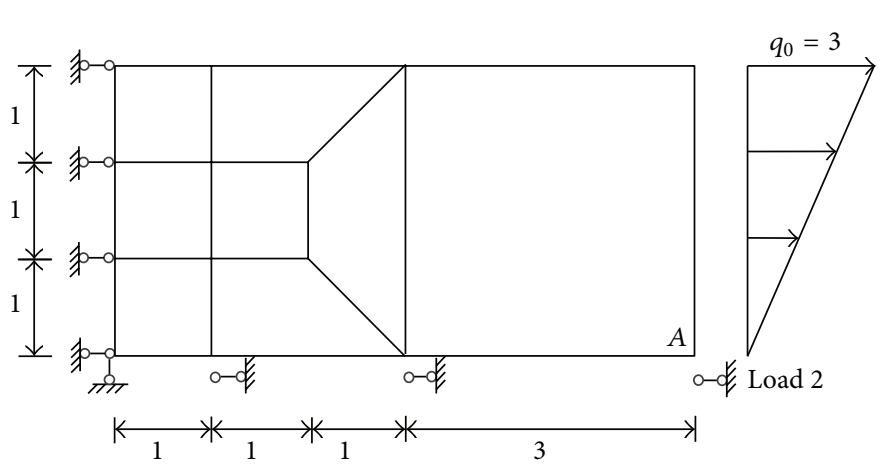

(b) Pure bending problem

FIGURE 13: A rectangular plate under two loading cases.

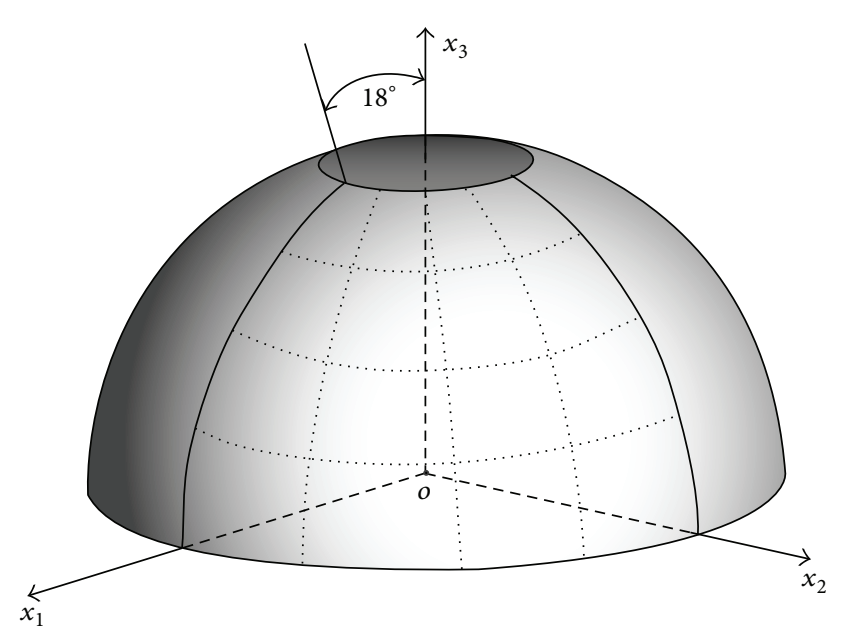

FIGURE 14: A hemispherical shell structure with an 18-degree hole on the top.

\section{Concluding Discussion}

In this paper a new method for establishing the local Cartesian coordinate system and calculating the derivatives of the shape function with respect to local coordinates is applied to the membrane elements GQ12 and GQ12M. When the membrane elements are introduced to be the component of the flat shell element, this new method can establish the

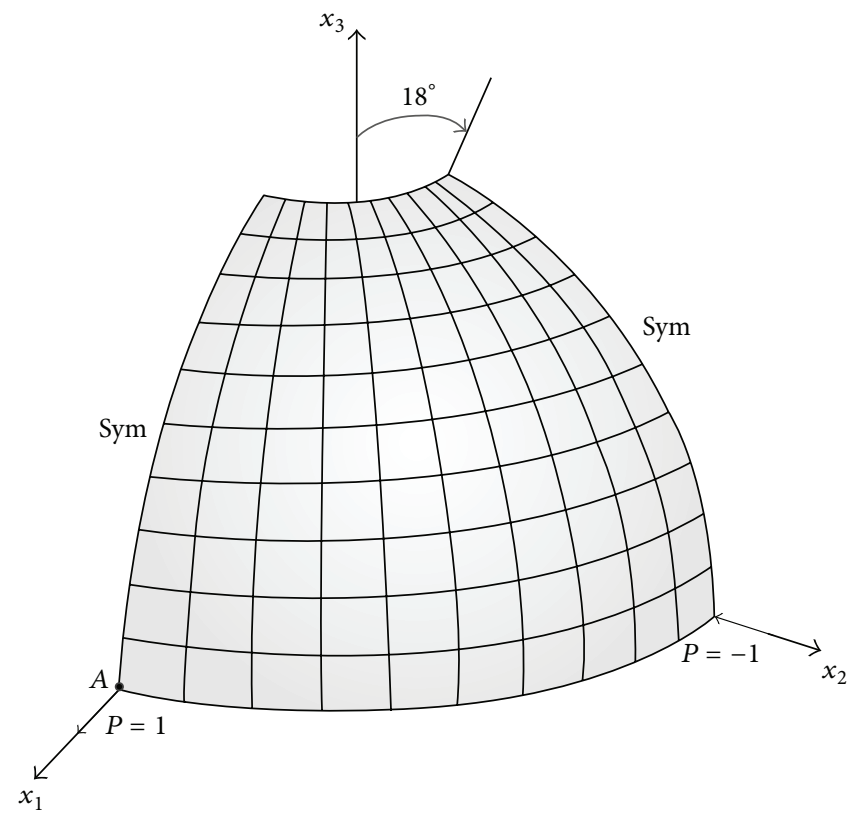

FIGURE 15: A quarter of the hemispherical shell structure with the meshes.

local Cartesian coordinate system on each Gauss point over the tangent plane to the element surface, and the precision is improved because the membrane elements are able to adapt the curved surface better. The numerical results of the test 
TABLE 6: The radial displacement at point A under different meshes.

\begin{tabular}{|c|c|c|c|c|}
\hline \multirow{2}{*}{ Flat shell element } & \multicolumn{4}{|c|}{ The radial displacement at $\mathrm{A}$ under different meshes $(\mathrm{m})$} \\
\hline & $4 \times 4$ & $8 \times 8$ & $12 \times 12$ & $16 \times 16$ \\
\hline Mindlin + GQ12 & 0.02234 & 0.05708 & 0.07222 & 0.08019 \\
\hline Mindlin + GQ12 (present method) & 0.03321 & 0.08975 & 0.09275 & 0.09329 \\
\hline Mindlin + GQ12M & 0.02365 & 0.06000 & 0.07470 & 0.08197 \\
\hline Mindlin + GQ12M (present method) & 0.03642 & 0.09027 & 0.09287 & 0.09335 \\
\hline Comparison solution [38] & \multicolumn{4}{|c|}{0.094} \\
\hline
\end{tabular}

problems show that the elements GQ12 and GQ12M with the new method can obtain comparatively high accuracies. This new method can bring some new ideas and approaches to improve the computing accuracies of other membrane elements and flat shell elements, so it has a good application prospect in the future.

\section{Conflict of Interests}

The authors declare that there is no conflict of interests regarding the publication of this paper.

\section{Acknowledgments}

The authors gratefully acknowledge the support by National Natural Science Foundation of China (11172055 and 11203040), China Postdoctoral Science Foundation (2014T70244), and Fundamental Research Funds for the Central Universities (DUT15RC(4)39).

\section{References}

[1] H. W. Zhang and K. P. Wang, "Multiscale methods for nonlinear analysis of composite materials," Acta Mechanica Sinica, vol. 36, no. 3, pp. 363-371, 2004.

[2] J. Lv, H. W. Zhang, and D. S. Yang, "Multiscale method for mechanical analysis of heterogeneous materials with polygonal microstructures," Mechanics of Materials, vol. 56, pp. 38-52, 2013.

[3] O. C. Zienkiewicz and R. L. Taylor, The Finite Element Method: Its Basis and Fundamentals, Beijing World Publishing, 2005.

[4] O. C. Zienkiewicz and R. L. Taylor, The Finite Element Method: Solid Mechanics, Butterworth-Heinemann, Oxford, UK, 2000.

[5] K. Kansara, Development of membrane, plate and flat shell elements in java [Ph.D. thesis], Virginia Tech, Blacksburg, Va, USA, 2004.

[6] G. P. Bazeley, Y. K. Cheung, B. M. Irons, and O. C. Zienkiewicz, "Triangular elements in plate bending-conforming and nonconforming solutions," in Proceedings of the Conference on Matrix Methods in Structural Mechanics, pp. 547-576, Wright Patterson AF Base, 1965.

[7] J. L. Batoz, K. J. Bathe, and L. W. Ho, "A study of three-node triangular plate bending elements," International Journal for Numerical Methods in Engineering, vol. 15, no. 12, pp. 1771-1812, 1980.

[8] J. L. Batoz and M. B. Tahar, "Evaluation of a new quadrilateral thin plate bending element," International Journal for Numerical Methods in Engineering, vol. 18, no. 11, pp. 1655-1677, 1982.
[9] J. L. Batoz, C. L. Zheng, and F. Hammadi, "Formulation and evaluation of new triangular, quadrilateral, pentagonal and hexagonal discrete Kirchhoff plate/shell elements," International Journal for Numerical Methods in Engineering, vol. 52, no. 5-6, pp. 615-630, 2001.

[10] M. M. Hrabok and T. M. Hrudey, "A review and catalogue of plate bending finite elements," Computers \& Structures, vol. 19, no. 3, pp. 479-495, 1984.

[11] M. Rezaiee-Pajand, F. Shahabian, and F. H. Tavakoli, "A new higher-order triangular plate bending element for the analysis of laminated composite and sandwich plates," Structural Engineering and Mechanics, vol. 43, no. 2, pp. 253-271, 2012.

[12] T. Nguyen-Thoi, P. Phung-Van, H. Luong-Van, H. NguyenVan, and H. Nguyen-Xuan, "A cell-based smoothed three-node Mindlin plate element (CS-MIN3) for static and free vibration analyses of plates," Computational Mechanics, vol. 51, no. 1, pp. 65-81, 2013.

[13] D. J. Allman, "A compatible triangular element including vertex rotations for plane elasticity analysis," Computers \& Structures, vol. 19, no. 1-2, pp. 1-8, 1984.

[14] P. G. Bergan and C. A. Felippa, "A triangular membrane element with rotational degrees of freedom," Computer Methods in Applied Mechanics and Engineering, vol. 50, no. 1, pp. 25-69, 1985.

[15] D. J. Allman, "A quadrilateral finite element including vertex rotations for plane elasticity analysis," International Journal for Numerical Methods in Engineering, vol. 26, no. 3, pp. 717-730, 2005.

[16] R. H. MacNeal and R. L. Harder, "A refined four-noded membrane element with rotational degrees of freedom," Computers \& Structures, vol. 28, no. 1, pp. 75-84, 1988.

[17] M. Iura and S. N. Atluri, "Formulation of a membrane finite element with drilling degrees of freedom," Computational Mechanics, vol. 9, no. 6, pp. 417-428, 1992.

[18] A. Cazzani and S. N. Atluri, "Four-noded mixed finite elements, using unsymmetric stresses, for linear analysis of membranes," Computational Mechanics, vol. 11, no. 4, pp. 229-251, 1993.

[19] R. Piltner and R. L. Taylor, "Triangular finite elements with rotational degrees of freedom and enhanced strain modes," Computers \& Structures, vol. 75, no. 4, pp. 361-368, 2000.

[20] S. Geyer and A. A. Groenwold, "Two hybrid stress membrane finite element families with drilling rotations," International Journal for Numerical Methods in Engineering, vol. 53, no. 3, pp. 583-601, 2002.

[21] A. A. Groenwold, Q. Z. Xiao, and N. J. Theron, "Accurate solution of traction free boundaries using hybrid stress membrane elements with drilling degrees of freedom," Computers \& Structures, vol. 82, no. 23-26, pp. 2071-2081, 2004. 
[22] N. Choi, Y. S. Choo, and B. C. Lee, "A hybrid Trefftz plane elasticity element with drilling degrees of freedom," Computer Methods in Applied Mechanics \& Engineering, vol. 195, no. 3336, pp. 4095-4105, 2006.

[23] S. Kugler, P. A. Fotiu, and J. Murin, "A highly efficient membrane finite element with drilling degrees of freedom," Acta Mechanica, vol. 213, no. 3, pp. 323-348, 2010.

[24] S. Cen, M.-J. Zhou, and X.-R. Fu, "A 4-node hybrid stressfunction (HS-F) plane element with drilling degrees of freedom less sensitive to severe mesh distortions," Computers \& Structures, vol. 89, no. 5-6, pp. 517-528, 2011.

[25] Y. Q. Long and Y. Xu, "Generalized conforming quadrilateral membrane element with vertex rigid rotational freedom," Computers \& Structures, vol. 52, no. 4, pp. 749-755, 1994.

[26] X.-W. Gao and T. G. Davies, Boundary Element Programming in Mechanics, Cambridge University Press, New York, London, UK, 2002.

[27] Y. F. Liu, J. Lv, R. X. Bai, and X. W. Gao, "A new method to compute thermal equivalent nodal loads on curved surfaces," Chinese Journal of Theoretical and Applied Mechanics, vol. 47, no. 2, pp. 328-336, 2015.

[28] J. H. Kane, Boundary Element Analysis in Engineering Continuum Mechanics, Prentice Hall, Englewood Cliffs, NJ, USA, 1994.

[29] P. Banerjee and T. Davies, "Advanced implementation of the boundary element methods for three-dimensional problems of elasto-plasticity," in Developments in Boundary Element Methods, Elsevier, London, UK, 1984.

[30] J. C. F. Telles and C. A. Brebbia, "On the application of the boundary element method to plasticity," Applied Mathematical Modelling, vol. 3, no. 6, pp. 466-470, 1979.

[31] T. A. Cruse, "An improved boundary-integral equation method for three dimensional elastic stress analysis," Computers \& Structures, vol. 4, no. 4, pp. 741-754, 1974.

[32] J. C. Lachat, A further development of the boundary integral technique for elastostatics [Ph.D. thesis], University of Southampton, Southampton, UK, 1975.

[33] A. A. Becker, The Boundary Element Method in Engineering, McGraw-Hill, London, UK, 1992.

[34] J. C. Lachat and J. O. Watson, "A second generation boundary integral program for three dimensional elastic analysis," in Boundary Integral Equation Method: Computational Applications in Applied Mechanics, T. A. Cruse and F. J. Rizzo, Eds., pp. 85-10, ASME, New York, NY, USA, 1975.

[35] Y. Q. Long and Y. Xu, "Generalized conforming triangular membrane element with vertex rigid rotational freedoms," Finite Elements in Analysis and Design, vol. 17, no. 4, pp. 259271, 1994.

[36] R. D. Cook, "Improved two-dimension finite element," Journal of the Structural Division, vol. 100, no. ST9, pp. 1771-1788, 1974.

[37] Y. Xu and Y. Q. Long, "A generalized conforming quadrilateral membrane element insensitive to geometric distortion," Acta Mechanica Sinica, vol. 13, no. 6, pp. 692-700, 1997.

[38] R. H. MacNeal and R. L. Harder, "A proposed standard set of problems to test finite element accuracy," Finite Elements in Analysis and Design, vol. 1, no. 1, pp. 3-20, 1985.

[39] L. Yu-qiu and H. Min-feng, "A generalized conforming isoparametric element," Applied Mathematics and Mechanics, vol. 9, no. 10, pp. 929-936, 1988.

[40] Y. X. Zhang and K. S. Kim, "Linear and geometrically nonlinear analysis of plates and shells by a new refined non-conforming triangular plate/shell element," Computational Mechanics, vol. 36, no. 5, pp. 331-342, 2005. 


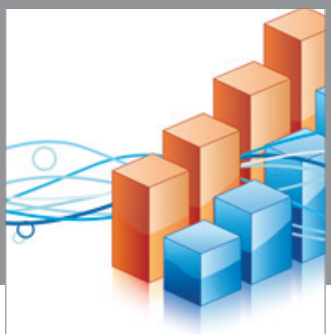

Advances in

Operations Research

vatem alat4

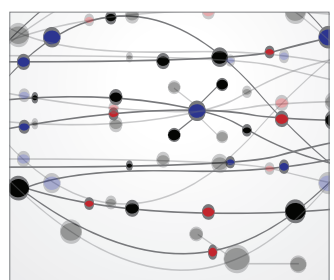

\section{The Scientific} World Journal
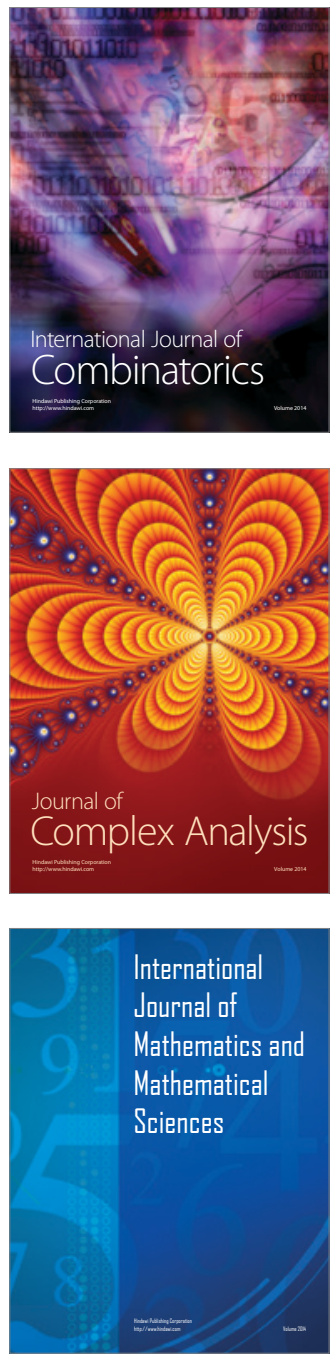
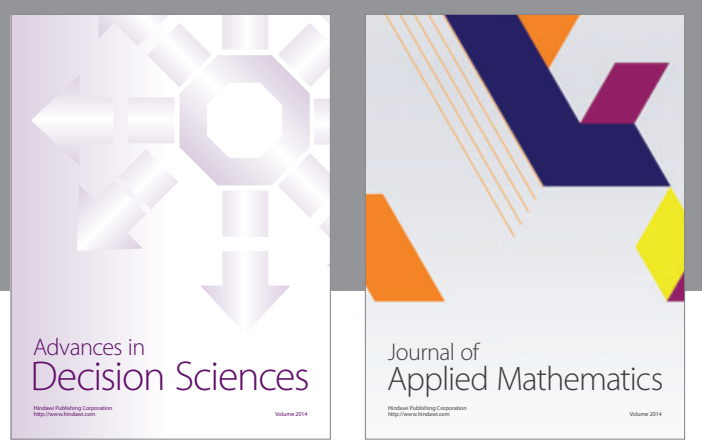

Algebra

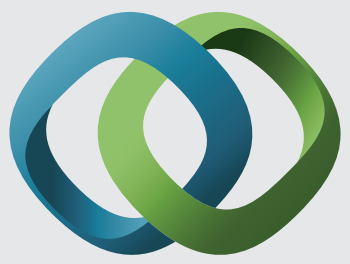

\section{Hindawi}

Submit your manuscripts at

http://www.hindawi.com
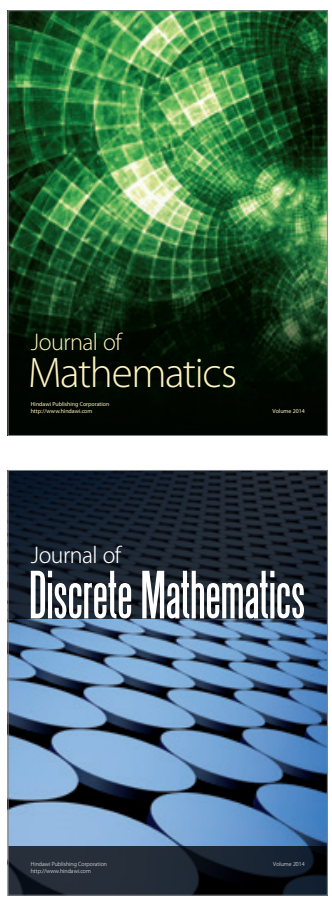

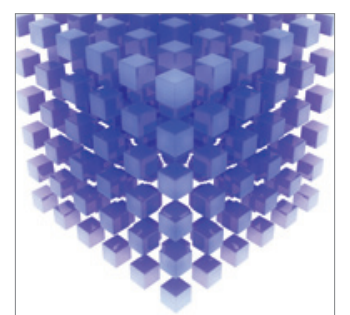

Mathematical Problems in Engineering
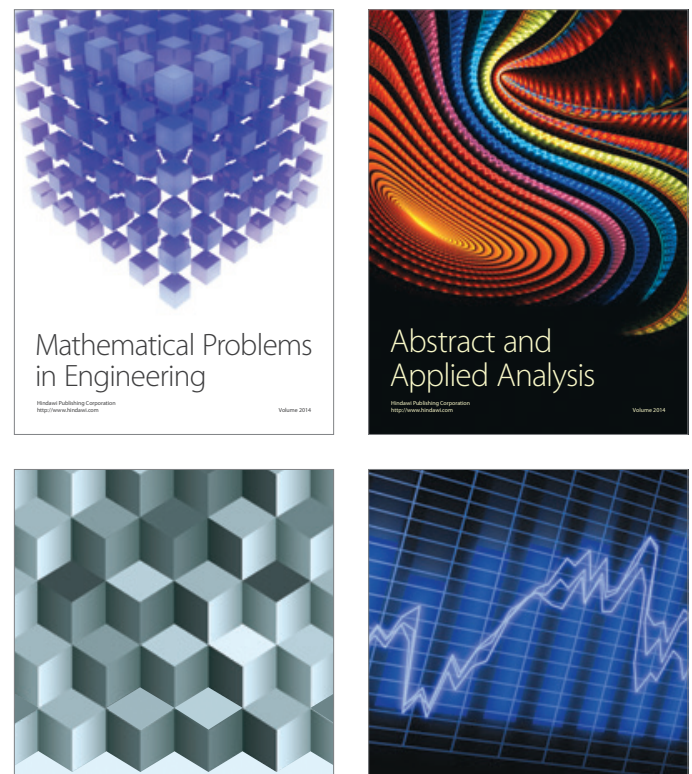

Journal of

Function Spaces

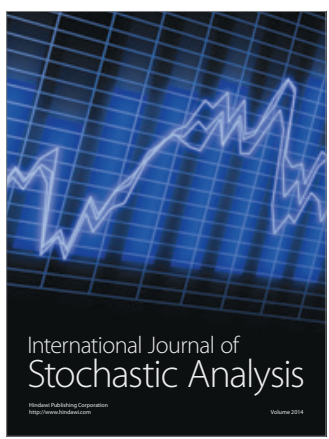

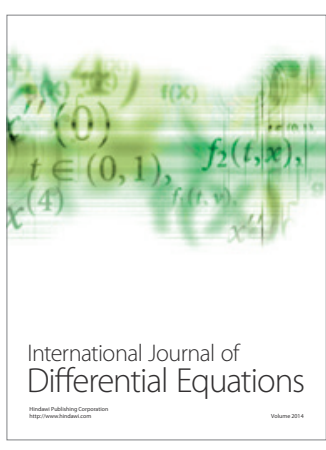
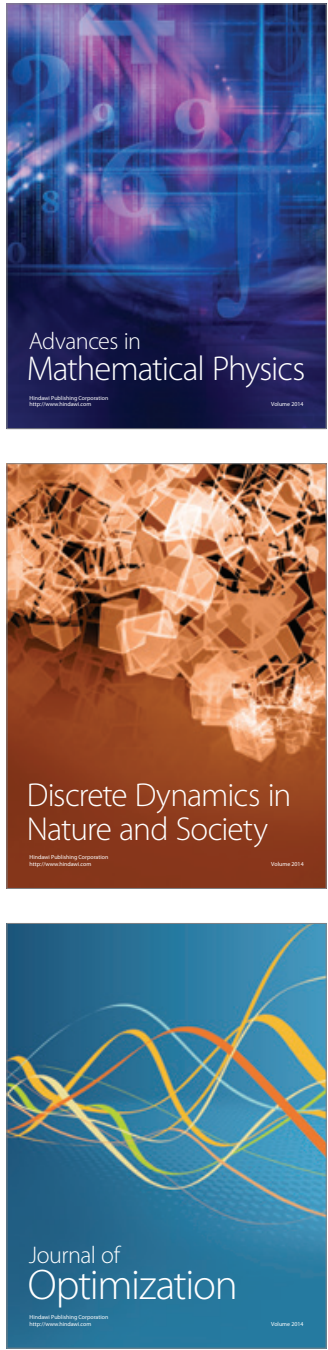